\title{
A Cardiac Asystole Case Presented With Seizure-Like
}

\section{Clinic}

\author{
Hikmet Saçmacı ${ }^{{ }^{\star}}$, Vahit Demir ${ }^{2}$, Tülin Kolusarı ${ }^{1}$, Nermin Tanık ${ }^{1}$, Emine Meşe ${ }^{1}$, Yasar Turan ${ }^{2}$ \\ ${ }^{1}$ Bozok University School of Medicine Department of Neurology, Yozgat \\ ${ }^{2}$ Bozok University School of Medicine Department of Cardiology, Yozgat
}

\begin{abstract}
The differential diagnosis of syncope and epilepsy appears as a very common entity in the emergency department. Conditions such as myoclonus or secondary seizure mimics may occur after cerebral hypoperfusion due to cardiac pathologies such as arrhythmia, asystole, atrioventricular block. Especially in non-recurrent interictal electrocardiography (EKG), pathologies that may be meaningful at all times may not be seen, and patients may be mistakenly referred to neurology as epilepsy. We wanted to present this case for the appropriate and necessary interventions to be carried out before it is too late in order not to increase potential mortality.

Key Words: Seizure-like conditions, cardiac asystole, electroencephalogram
\end{abstract}

\section{Introduction}

Recurrent episodes of unconsciousness and seizurelike conditions are common clinical disorders frequently encountered by neurologists (1). Cardiac arrhythmias, syncope and non-epileptic psychogenic seizures are among the differential diagnoses; moreover, all these diseases require accurate diagnosis and rapidly treatment (2). Also it is known that cardiac asystole leads to clinical symptoms similar to seizures (3). Vagal-mediated short cardiac asystole leads to transient cerebral ischemia and may suggested clinical findings and seizure-like manifestations (4). The differential diagnosis of neurocardiogenic syncope and partial seizures associated with secondary generalization cannot be made with great accuracy based solely on the anamnesis; since interictal electroencephalogram (EEG) is generally normal, video EEG monitoring, brain imaging and cardiac evaluation may be required for the certain diagnosis (5).

Herein, we describe to raise awareness due to the risk of the cardiac case, who applied to the emergency service with the complaints of sudden change in consciousness, hesitations and contractions, to be diagnosed with epileptic seizures.

\section{Case Report}

The 63-year-old male patient with no history of epilepsy was evaluated in the emergency service due to sudden contraction attacks beginning in both arms and then spreading over the whole body and staring blankly for one week. The patient's complaints were repeated 8-10 times a day and continued for 4-8 seconds; afterwards, he had drowsiness and weakness. EEG was performed on the patient who was hospitalized upon the normal detection of his laboratory and electrographic tests, brain tomography and magnetic resonance imaging (MRI) tests. His neurological examination was within the normal limits and no pathological finding was encountered on the patient's EEG (Fig.1). Since these contraction attacks continued in the follow-ups of the patient, $2 \times 500 \mathrm{mg}$ levetiracetam therapy started to be given, and no decrease was observed in his symptoms after the treatment. Cardiology was consulted for the differential diagnosis of cardiac syncope and holter monitoring revealed complete AV blocks with duration of 8-10 seconds (Fig.2) although the patient's basal electrocardiography was normal. Levetiracetam was stopped and a pacemaker was placed in the patient for whom a cardiac pacemaker was recommended. It was observed that there were no similar complaints during the check up after 3 months.

\section{Discussion}

Here, we have presented the case that appears like a seizure due to cardiac asystole. The patient had repeated short-term sudden rhythmic contraction movements in the emergency room and follow-ups,

${ }^{*}$ Corresponding Author: Hikmet Saçmacı, Bozok University School of Medicine-Department of Neurology-Yozgat-Turkey E-mail: hsacmaci@hotmail.com

ORCID ID: Hikmet Saçmacı: 0000-0003-1480-0562, Vahit Demir: 0000-0001-8349-6651, Tülin Aktürk: 0000-0003-1818-1578, Nermin Tanık: 0000-0002-7567-9244, Emine Meşe: 0000-0002-2216-946X, Yasar Turan: 0000-0002-2796-899X 


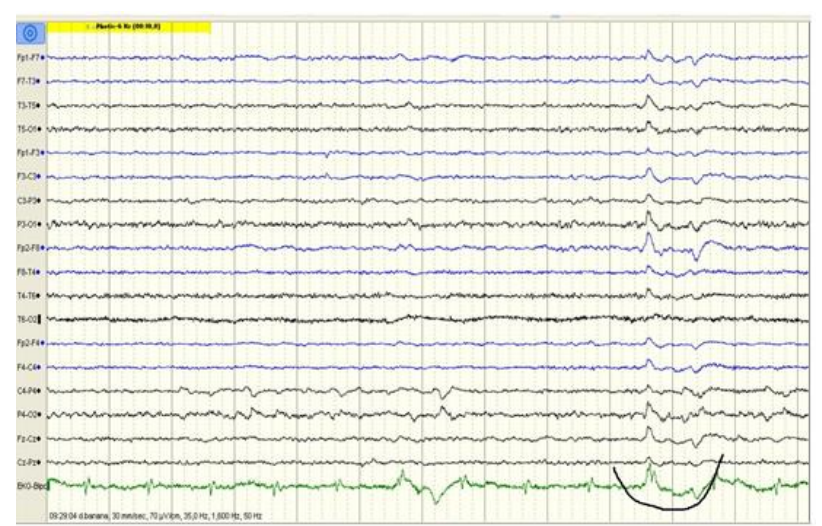

Fig. 1. Negative scalp EEG, including sleep recordings using "double banana montage" Low filter: $0.3 \mathrm{~Hz}$; highfilter: $35 \mathrm{~Hz}$; notch filter: $50 \mathrm{~Hz}$; time base: 30 $\mathrm{mm} /$ second; sensitivity: $70 \mu \mathrm{V} / \mathrm{cm}$

and anti-epileptic treatment was initiated until the detailed cardiology examination was performed because the EKG in the interictal period did not show any signs of asystole, and this disturbances repeated many times during the day. The patient has no aura findings before in terms of semiology, and myoclonic jerks occurring after the tonic contraction and the tendency to sleep afterwards so these complaints have been supported the possibility of epilepsy as the first diagnosis. Furthermore, a slight paroxysmal disturbance in the patient's short EEG indicates after photic stimulation and the absence of abnormal findings in concomitant EKG reinforced this possibility. Jackson et al., in their studies, which included asystole presenting as seizures, defined seizure semiology in 3 stages as the preasystole period, in which there is a general weakness, the asystole period in which cerebral hypoperfusion occurs which is followed by the cerebral reperfusion period (1). However, diffuse deceleration in the myoclonia stage in EEG shots and the asystole on EKG are different aspects of our case. During the bradycardia and early asystole, patients developed a distinctive rapid breathing pattern and our patient was observed to have a weakness state. In general, the findings observed in asystole-induced seizures are summarized in the literature. During seizures, there may also be a temporary pallor and slight dizziness and atypical chest pain. A general pallor at the onset of sinus bradycardia associated with hypoperfusion during the asystole and tonic contraction during the asystole are the conditions stated in the literature (6). During seizures, there may also be a temporary pallor and slight dizziness and atypical chest pain (7). Diffuse EEG deceleration in sinus bradycardia and early asystole is among the expected findings (7). It is

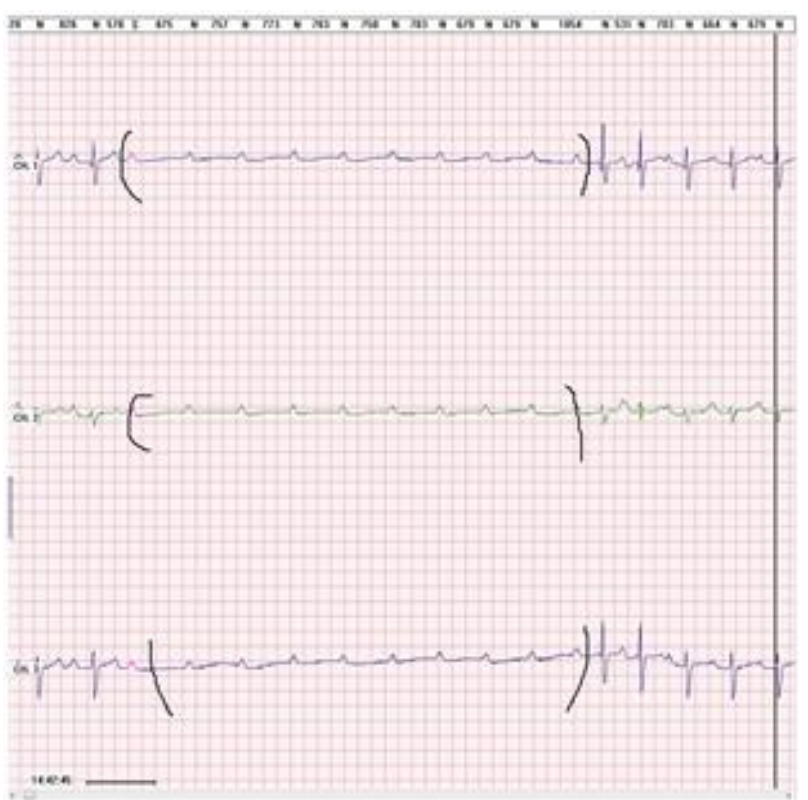

Fig. 2. Monitor strip revealing asystole recording on the Holter

assumed that the inhibitory effects of hypoxia at the cortical level are responsible for the pathophysiology of the seizure-like activity. As a result of this inhibition, tonic contractions, which especially result from the brainstem reticular formation level, may occur and teta and delta waves may appear in EEG (1). In our patient, no EEG findings occurred during 4-8 sec asystole. As Gestaut noted, while EEG abnormalities may not be detected in asystole lasting less than 6 seconds, slow waves appeared in the EEG during the cardiac arrest lasting between 7 and 13 seconds (8). We wanted to present the case because cardiac asystole is a clinical entity with high mortality and morbidity when it is not intervened on time. Also there are a limited number of case samples in the literature and the asystole cases who have seizure-like contractions are confused with epileptic seizures.

We conclude that differential diagnosis should be made quickly and in detail for patients presenting with seizure-like movement disorder.

Acknowledgements: We thank our patient for their contributions to the study.

Ethics Informed Consent: Consent form was filled out by all participants. Peer-review: Internally peerreviewed.

Authorship Contributions: Concept: H.S., T.A., Design: H.S., T.A., V.D., Data Collection or Processing: H.S., T.A., V.D.,N.T., Y.T., Analysis or Interpretation: H.S., T.A., V.D.,N.T., Y.T., Literature Search: H.S., Writing: H.S., T.A.,

Conflict of Interest: No conflict of interest was declared by the authors. 
Financial Disclosure: The authors declared that this study received no financial support

\section{References}

1. Jackson A, Bower S, Seneviratne U. Semiologic, electroencephalographic and electrocardiographic correlates of seizure-like manifestations caused by cardiac asystole. Seizure 2015; 29: 15-19.

2. Jallon P. Arrhythmogenic seizures. Epilepsia 1997; 38: 43-47.

3. Andriola M, Editor Pseudo-Seizures Secondary To Cardiac Asystole And Apnea. Electroencephalography And Clinical Neurophysiology; 1983: Elsevier Sci Ireland Ltd Customer Relations Manager, Bay 15, Shannon
4. Cebe L, Singh H. Reflex anoxic seizures (RAS) in an adult patient: a separate entity from epilepsy. Case Reports 2018; 2018: 2017-222389.

5. Gavris MF, Khalighi K, Mascarenhas DA. Idiopathic cardiac asystole presenting as epileptic seizures. Journal of electrocardiology 2002; 35 : 279-283.

6. $\mathrm{Xu} \mathrm{Z}$, Bower S, Seneviratne U. Severe cardioinhibitory vasovagal syncope in sleep and supine posture. Epileptic Disorders 2014; 16: 101 106.

7. Venkataraman V, Wheless JW, Willmore L, Motookal H. Idiopathic cardiac asystole presenting as an intractable adult onset partial seizure disorder. Seizure 2001; 10: 359-364.

8. Gastaut H. Syncope and seizure. Electroencephalography and Clinical Neurophysiology 1958; 10: 571-572. 